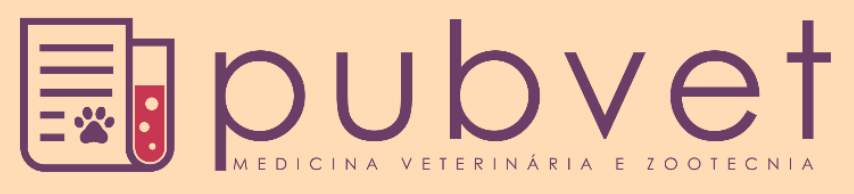

https://doi.org/10.31533/pubvet.v14n8a628.1-11

\title{
Bem-estar de bezerros durante o aleitamento e a desmama em diferentes sistemas de criação: Revisão
}

\author{
Lucyelen Costa Amorim Pereira ${ }^{1 *} \bullet$, Aparecida de Fatima Madella-Oliveira ${ }^{2} \bullet$ \\ ${ }^{1}$ Mestranda em Agroecologia do Instituto Federal do Espirito Santo - campus de Alegre, Brasil. \\ ${ }^{2}$ Professora do Instituto Federal do Espírito Santo - campus de Alegre, Alegre, ES, Brasil. \\ *Autor para correspondência, E-mail: lucyelen_amorim@hotmail.com.br
}

Resumo. O estado em que o animal se encontra interfere diretamente no grau do seu bemestar, comprometendo a produção e a qualidade dos produtos de origem animal. Nesse sentido, a busca pelo mercado por produtos de qualidade e que visem o bem-estar dos animais tem feito com que produtores busquem formas de manejo alternativos como por exemplo, o modo como os animais são alojados. Animais quando alojados de forma inadequada são impedidos de realizarem comportamentos normais da espécie. Quando a instalação não favorece interações seja com o ambiente, seja com outros animais podem ocorrer as estereotipias. Tal comportamento pode indicar que o animal está tendo um bemestar pobre e consequentemente esses fatores irão interferir na qualidade da produção. Portanto, o objetivo do presente trabalho é fazer uma revisão de literatura, abordando a importância do bem-estar para bezerros durante o aleitamento e a desmama submetidos a diferentes sistemas de criação.

Palavras chave: Bovinos, instalação, sistema tropical

\section{Calf well-being during lactation and weaning in different rearing systems: Review}

Abstract. The state in which the animal is found directly interferes with the degree of its well-being, compromising the production and quality of products of animal origin. In this sense, the search by the market for quality products that aim at the welfare of animals has led producers to seek alternative ways of handling such as, for example, the as animals are housed. Animals, when improperly housed, are prevented from performing normal behaviors of the species. When the installation does not favor interactions either with the environment or with other animals, stereotypies may occur. Such behavior can indicate that the animal is having a poor welfare and consequently these factors will interfere with the quality of production. Therefore, the objective of the present work is to realize a literature review, addressing the importance of welfare for calves during lactation and weaning submitted to different rearing systems.

Keywords: Cattle, installation, tropical system

\section{Bienestar de los terneros durante la lactancia y el destete en diferentes sistemas de cría: Revisión}

Resumen. El estado en el que se encuentra el animal interfiere directamente con el grado de su bienestar, comprometiendo la producción y la calidad de los productos de origen animal. En este sentido, la búsqueda en el mercado de productos de calidad que tengan como objetivo el bienestar de los animales ha llevado a los productores a buscar formas 
alternativas de manipulación, como, por ejemplo, la forma en que se alojan los animales. Los animales, cuando se alojan incorrectamente, no pueden realizar comportamientos normales de la especie. Cuando la instalación no favorece las interacciones con el medio ambiente o con otros animales, pueden producirse estereotipos. Tal comportamiento puede indicar que el animal está teniendo un malestar y, en consecuencia, estos factores interferirán con la calidad de la producción. Por lo tanto, el objetivo del presente trabajo es hacer una revisión de la literatura, abordando la importancia del bienestar de los terneros durante la lactancia y el destete sometidos a diferentes sistemas de cría.

Palabras clave: Ganado, instalación, sistema tropical

\section{Introdução}

As preocupações do produtor em relação à qualidade de vida dos animais de fazenda são frequentemente focadas em práticas específicas, como a separação da vaca e do bezerro imediatamente após o nascimento. Separar o bezerro da vaca logo após o nascimento é uma prática rotineira em fazendas leiteiras em todo o mundo (Busch et al., 2017). Estes mesmos autores relatam que os defensores dessa prática argumentam que ela impede que a vaca e o bezerro formem uma ligação forte e, portanto, reduz o estresse associado à separação quando ela ocorre (Busch et al., 2017).

$\mathrm{O}$ estado em que o animal se encontra interfere diretamente no grau do seu bem-estar, comprometendo a produção e a qualidade dos produtos de origem animal. Nesse sentido, a eficiência de uma instalação está diretamente relacionada à qualidade do ambiente e o grau de conforto que ela pode proporcionar aos animais (Campos \& Campos, 2004).

O sistema de criação deve ser por aquele capaz de expressar ao máximo o potencial genético do animal. Oliveira \& Azevedo (2005) abordam sobre o sistema coletivo de criação, sendo aquele em que o animal permanece com a mãe até a desmama natural. De acordo com os autores, é um sistema utilizado geralmente em rebanhos leiteiros muito pouco tecnificados, ou ainda em rebanhos compostos basicamente por vacas zebuínas, principalmente da raça Gir, onde o produtor acredita que as fêmeas somente produzirão na em presença da cria. Geralmente esse sistema é utilizado em rebanhos de pouca especialização e de baixa expectativa de produção.

Os sistemas do tipo casinha e tropical surgiram como uma boa alternativa, representando um avanço em relação aos bezerreiros coletivos. Contudo, é imprescindível que o sistema de criação adotado, seja de baixo custo, rentável, que proporcione bem-estar e permita que o animal expresse todo seu potencial genético.

Há uma preocupação com o comportamento de animais que são criados de forma individual sem nenhum contato social. Animais que são criados em grupo são mais ativos (Oliveira \& Azevedo, 2005). Vieira \& Shields (2016) salientam que a socialização pode incentivar a ingestão de alimentos sólidos mais cedo. De acordo com os autores, os animais ao observarem outros animais, começarão a comer o mesmo alimento permitindo uma transição alimentar mais tranquila.

Animais de produção quando submetidos ao intensivo confinamento podem ser frustrados e estressados. Quando os estímulos naturais proporcionados pelo ambiente são restritos, favorecem o surgimento das estereotipias e prejudicam o desenvolvimento físico dos animais (Broom \& Molento, 2004).

Um animal encontra-se em bom estado de bem-estar, quando está saudável, seguro, confortável, bem nutrido e capaz de expressar comportamentos naturais. Em contrapartida, estados desconfortáveis como dor, medo e angústia representam um bem-estar ruim (Broom \& Molento, 2004). O bem-estar ruim de um animal provavelmente está relacionado com o empobrecimento ambiental e com as frustrações as quais o animal encontra-se submetido. Dessa forma, tornar o ambiente complexo e estimular a expressão de comportamentos naturais podem ser alcançados por meio da adoção de técnicas de enriquecimento ambiental (Costa et al., 2012).

Uma forma de identificação de alguns sinais de bem-estar precário é a mensuração fisiológica, como por exemplo o aumento na frequência cardíaca e respostas imunológicas reduzidas após um desafio (Broom \& Molento, 2004). 
A busca pelo mercado por produtos de qualidade e que visem o bem-estar dos animais tem feito com que produtores busquem formas de manejo alternativos. Esse progresso na produção de animais torna necessário o estabelecimento de critérios que avaliem o bem-estar em sistemas de criação. Portanto, é importante caracterizar padrões comportamentais e de alimentação de bezerros leiteiros, pois está relacionado à saúde e ao bem-estar dos mesmos. Estabelecer esses padrões torna-se relevante na tomada de decisão quanto à nutrição e gestão (Carneiro, 2015).

Deste modo, o presente trabalho objetivou-se fazer uma revisão de literatura, abordando a importância do bem-estar para bezerros durante o aleitamento e a desmama em diferentes sistemas de criação.

\section{Bem-estar animal}

Bem-estar animal é um conceito que diz respeito à qualidade de vida que o animal experimenta, principalmente as experiências boas e prazerosas. A visão do homem sobre os animais mudou, ao longo da história, de acordo com o grau de conhecimento vigente na época. Inicialmente, nos séculos XVI e XVII pensava-se que os animais eram máquinas e não tinham mente, já que não eram capazes de falar ou pensar (Gonçalves et al., 2010).

O bem-estar de um indivíduo é seu estado em relação às suas tentativas de adaptar-se ao seu ambiente (Broom, 1986). O conceito de bem-estar refere-se ao estado de um indivíduo em uma escala variando de muito bom a muito ruim (Broom \& Molento, 2004). De forma geral, o conceito de bem-estar baseiase nos princípios das cinco liberdades instituídas pelo FAWC (2009). De acordo com as cinco liberdades os animais devem estar: 1) Livres de fome, sede e desnutrição; 2) Livres de desconforto; 3) Livres de dor, ferimentos e doenças; 4) Livres para expressar seu comportamento; 5) Livres de medo e estresse (Filipini et al., 2016).

O bem-estar animal é o "estado de harmonia entre o animal e seu ambiente, caracterizado por condições físicas e fisiológicas ótimas e alta qualidade de vida do animal" (Kondo \& Hurnik, 1988).

O estresse fisiológico é um dos principais indicadores usados na avaliação do bem-estar animal. Estresse pode, de maneira geral, ser considerado a resposta fisiológica do organismo a um estímulo do ambiente, na tentativa de manter a homeostasia (Hötzel \& Machado Filho, 2004). O estresse pode ainda interferir com a memória dos animais e o acarretar uma menor capacidade cognitiva. Isto, por sua vez, pode gerar comportamentos inapropriados e afetar negativamente o bem-estar (Mendl, 2001).

De acordo com Broom \& Molento (2004) um animal que se encontra impossibilitado de adotar uma postura preferida de repouso, seu bem-estar é considerado como pobre, ou seja, comportamentos anormais, tais como estereotipias, automutilação ou comportamento excessivamente agressivo indicam condições de baixo grau de bem-estar.

O comportamento estereotipado é classificado como um dos tipos de comportamento anormal (Fraser \& Broom, 1997). Segundo Miranda-de la Lama et al. (2012) estereotipias podem ser definidas como comportamentos repetitivos, invariantes e sem uma função ou objetivo óbvio. Além disso, pode representar uma compensação desenvolvida pelo animal, com a finalidade de diminuir as tensões e ou frustrações, quando exposto a um ambiente com baixo bem-estar (Lauber et al., 2006). Em sistemas intensivos onde o espaço é reduzido e ocorre o constante reagrupamento de animais, ocorre o aumento da agressividade, porque os animais têm de competir mais para se alimentar e disputar lugares para deitar. Sob estas condições, alguns indivíduos estarão mais bem sucedidos do que outros no acesso à alimentação ou outros recursos (Galindo et al., 2000).

\section{Aleitamento e desmama}

Os três primeiros meses de vida dos bezerros são os mais críticos, pois o seu sistema imunológico ainda não se encontra totalmente desenvolvido. Após o nascimento o colostro é a principal fonte de proteção contra os microrganismos patogênicos presentes no ambiente (Alvim et al., 2005). Além de fornecer anticorpos, o colostro apresenta um alto valor nutritivo e estimula as funções normais do trato digestivo (Rodríguez et al., 2009; Santos et al., 1988, 1994).

Paranhos da Costa \& Cromberg (1997) definem o aleitamento natural como sendo aquele em que os bezerros mamam diretamente nas mães, permanecendo com elas após as ordenhas. De acordo com os 
autores, este tipo de aleitamento é adotado em rebanhos nos quais as vacas não são capazes de manter a lactação sem a presença do bezerro. Os mesmos autores relatam que nesse tipo de aleitamento é difícil controlar a quantidade de leite ingerida, e por isso, é importante que haja o monitoramento por parte do produtor para assegurar de que o animal esteja ingerindo a quantidade adequada de leite.

No aleitamento natural, a ocorrência de distúrbios gastrointestinais diminui uma vez que, os bezerros obtêm o leite diretamente do teto ingerindo-o mais limpo. Além disso, estudos mostram que vacas mestiças, com potencial de $3.500 \mathrm{~kg}$ de leite/lactação, produzem $10 \%$ a mais de leite comercializável com o bezerro ao pé, que aquelas cujos bezerros foram apartados ao nascer (Campos \& Campos, 2004). Paranhos da Costa (2004) também discutem o conceito de aleitamento artificial, que consiste no fornecimento do leite ou de substitutos de leite em baldes ou em mamadeiras sendo realizado em propriedades de gado especializado para produção leiteira. As vantagens do aleitamento artificial são o controle da quantidade de leite fornecida, bem como ordenhas mais higiênicas e mais rápidas (Miranda et al., 2003).

Como o leite é um alimento nobre e o principal produto gerador de renda da propriedade leiteira, é desejável que ele seja substituído por uma dieta de menor custo (Oliveira et al., 2013). Uma alternativa ao aleitamento convencional é o aleitamento fracionado (Vieira \& Silva, 2014). O aleitamento fracionado consiste no fornecimento da dieta líquida acima de $10 \%$ do peso corporal por bezerro nas semanas iniciais de vida, com posteriores reduções antes do desaleitamento, com o objetivo de estimular o consumo de matéria seca pelos animais (Prado \& Von Tiesenhauser, 1987, 1988). A adoção de aleitamento fracionado pode permitir que os bezerros recebam quantidade de dieta líquida mais próxima ao que teriam em sistema com acesso ad libitum nas primeiras semanas de vida (Jasper \& Weary, 2002; Passillé et al., 2008).

No aleitamento fracionado, a redução na oferta de leite pode ser iniciada quando os bezerros atingirem, aproximadamente 20 dias de idade, desde que apresentem boas condições de saúde. Já no aleitamento convencional, como não é possível controlar exatamente quanto o bezerro está mamando, recomenda-se fazer a mudança monitorando seu comportamento como, por exemplo, a sua vocalização e ingestão de ração (Vieira \& Silva, 2014). Em um trabalho realizado por Azevedo et al. (2014) comparando o sistema de aleitamento fracionado com o aleitamento convencional, os autores observaram que os dois sistemas apresentaram resultados similares com relação ao ganho de peso e incidência de diarreias. Entretanto, o sistema de aleitamento fracionado possibilitou maior consumo total de matéria seca e os animais mantidos nesse sistema apresentaram menor custo por kg de peso ganho.

A amamentação é um dos fatores que influencia a retomada da atividade sexual pós-parto em vacas de corte (Moura et al., 2014). A presença do bezerro ao pé da vaca e o ato de mamar criam uma série de estímulos metabólicos, nervosos e fisiológicos, os quais podem impedir a vaca de retomar o seu ciclo estral (Williams, 2005). A utilização do desmame dos bezerros a idades precoces (60-110 dias) mostrase eficiente em incrementar a fertilidade das vacas (Almeida et al., 2002). Todavia, Moura et al. (2014) salientam que não se deve deixar de considerar o desempenho ponderal de bezerros, os mesmos não podem ter seu crescimento comprometido, para não afetar o tempo e a idade de acabamento e terminação dos machos e também a idade de acasalamento das fêmeas. Restle et al. (2003) afirmam que o principal objetivo do desmame precoce não é promover no bezerro ganho de peso maior aos obtidos ao pé da vaca, mas incrementar um ganho mínimo que não prejudique seu desempenho futuro.

\section{Sistemas de criação}

Os objetivos gerais das instalações para bezerros são a proteção dos extremos térmicos e climáticos, acesso adequado ao alimento, garantia da segurança no que diz respeito a ferimentos e controle da saúde e o bem-estar dos bezerros (Bittar, 2016).

O uso contínuo de uma mesma instalação pode resultar em maiores gastos com medicamentos e menores ganhos de peso dos animais. Pellegrini et al. (2006) recomendam-se algumas instalações durante a fase de aleitamento, como a criação a pasto, uso de abrigos as chamadas "casinhas" e o uso de telas de sombreamento, chamado de sistema tropical. Os sistemas mais utilizados para criação de bezerras baseiam-se no alojamento individual ou em grupo. Oliveira et al. (2014) descreveram uma versão da criação individual, mais adaptada às condições climáticas do país e mão-de-obra, chamado de 
sistema argentino ou sistema tropical que consiste em apresentar custos relativamente mais baixos se comparado às "casinhas" tradicionais.

\section{Sistema tropical}

Neste sistema, os animais são alojados individualmente, contidos por uma coleira e corrente $(1,5$ metros) a um fio de aço de aproximadamente 12 metros (Campos \& Campos, 2004) (Figura 1). O animal tem uma área mais ampla e possibilidade de escolha do local para se deitar, além do controle da nutrição também ser individualizado (Bittar, 2016). Uma pesquisa conduzida no Campo Experimental Santa Mônica, da Embrapa Gado de leite evidenciou que o uso de telas de sombreamento proporcionou bom desempenho dos animais, com resultados semelhantes aos mantidos em abrigos individuais. Os dois sistemas possibilitam o consumo mais precoce do concentrado, resultando em maior ganho de peso e facilitando a socialização após o desaleitamento (Campos \& Campos, 2004). Esse sistema tem como finalidade substituir outros sistemas de criação, pois constitui uma opção mais econômica e de fácil instalação (Silva, 2018).
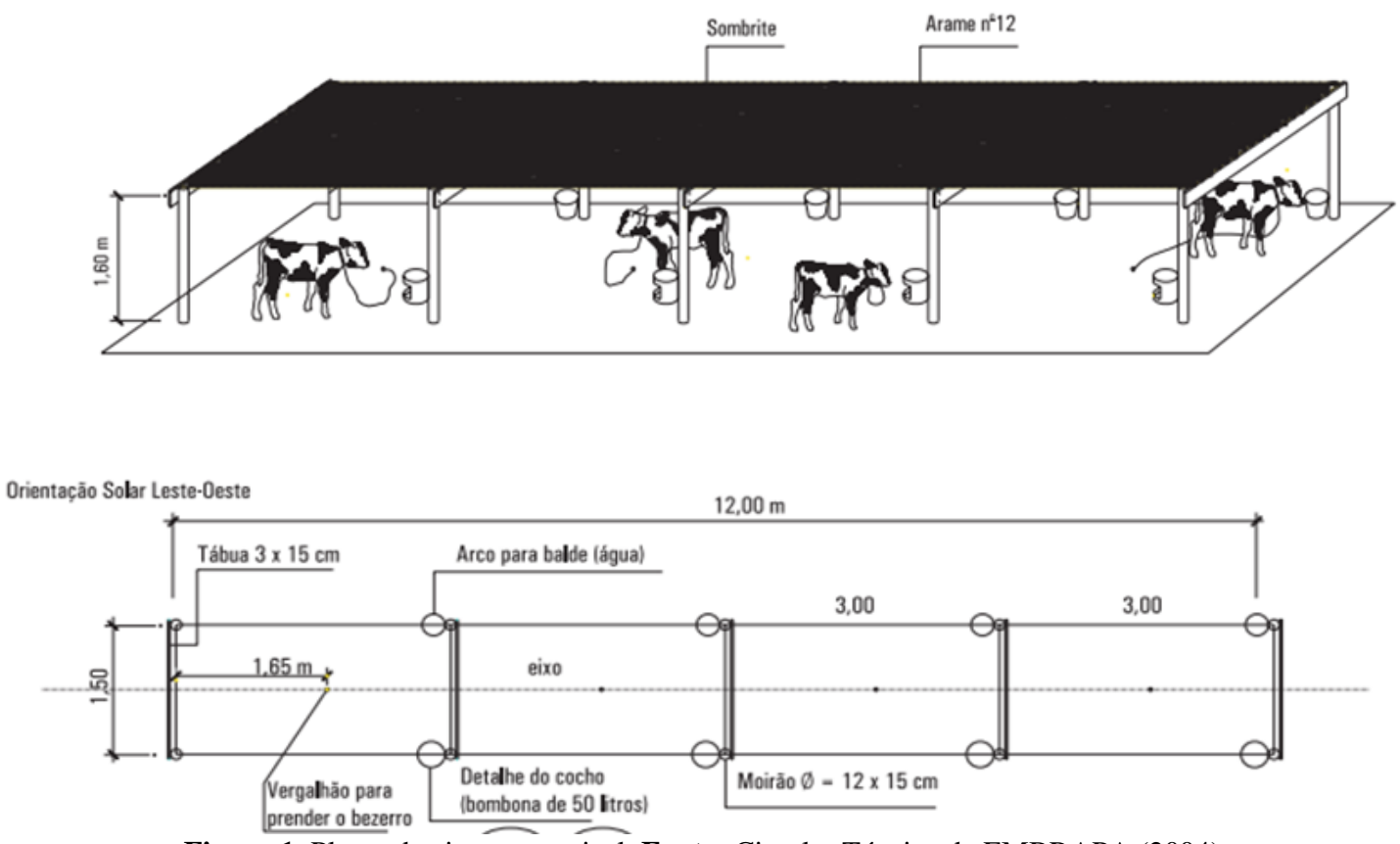

Figura 1. Planta do sistema tropical. Fonte: Circular Técnica da EMBRAPA (2004).

\section{Sistema de casinhas}

A criação em casinhas consiste no alojamento dos bezerros com acesso a um pequeno abrigo individual ao ar livre. Neste tipo de criação os bezerros têm mobilidade limitada, uma vez que ficam amarrados, com acesso a casinha e a uma pequena área externa Paranhos da Costa \& Cromberg (1997) (Figura 2). O sistema de criação em casinhas apresenta como vantagens a diminuição do contato e, consequentemente, a minimização da transmissão de doenças entre os animais; o acompanhamento clínico individual, o que facilita a observação de qualquer problema de saúde, além de não existir competição entre os animais (Oliveira \& Azevedo, 2005). Devido aos bons resultados apresentados, o uso desse sistema tem aumentado, proporcionando menor problema sanitário, menor mortalidade e promovendo um desejável aumento no consumo de concentrados e volumosos (Gomes \& Madureira, 2016).

No entanto, alguns estudos relatam que quando os animais não estão agrupados, são privados de interação social e de espaço para exercitar-se podendo dessa forma, comprometer seu bem-estar e consequentemente seu desenvolvimento (Bittar, 2016).

Quando o sistema de criação dispõe de espaço limitado, os animais apresentam comportamento de movimento locomotor-rotacional, substituindo ocasionalmente esse comportamento por coices ou pulos (Façanha et al., 2011). De acordo com Broom \& Molento (2004), quando os estímulos naturais proporcionados pelo ambiente são restritos, favorecem o surgimento das estereotipias e prejudicam o 
desenvolvimento físico dos animais. Quando criados em baias ou gaiolas muito pequenas, ou gaiolas metabólicas, os bezerros tentam limpar-se exaustivamente. Porém, esse comportamento torna-se impossível devido à restrição de espaço no lugar os quais foram inseridos.

Animais de produção intensivamente confinados podem ser frustrados e estressados. Os alojamentos individuais impossibilitam a expressão dos comportamentos naturais, com exceção de deitar-se, levantar-se e dar poucos passos (Vieira \& Silva, 2014).

O sistema de casinha tem por principal objetivo a redução na disseminação de doenças (Bittar, 2016). Entretanto, esse tipo de instalação impede os bezerros de expressarem seu comportamento natural como brincar, correr e até mesmo cuidar um do outro (Campos \& Campos, 2004; Oliveira \& Azevedo, 2005; Vieira \& Silva, 2014).

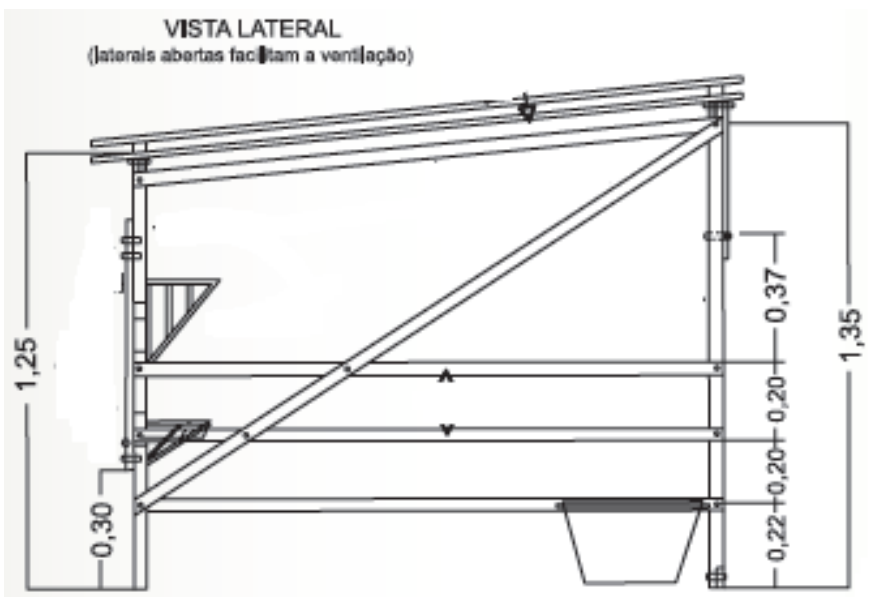

Figura 2. Sistema de casinha. Fonte: Circular Técnica da EMBRAPA (2005).

\section{Sistema coletivo}

A criação de bezerras em sistemas coletivos baseia-se no princípio de que os bezerros leiteiros são animais com hábitos gregários e o alojamento em grupo permite o desenvolvimento de comportamento social (Bittar, 2016). O alojamento coletivo permite a manifestação de comportamentos lúdicos, ou seja, o exercício e o jogo entre bezerros dentro do grupo. Assim, a criação de animais em lotes tem sido considerada a mais adequada, do ponto de vista do bem-estar e do comportamento animal, por alguns pesquisadores ( Bittar, 2016).

Preocupações com bem-estar dos animais tem sugerido o agrupamento de bezerras em pequenos lotes para que ocorra socialização entre os animais reduzindo assim o estresse (Gomes \& Madureira, 2016). Entretanto, este sistema parece mais propício à propagação de doenças (Burguer et al., 2000; Carvalho et al., 2012). O contato social com a mãe e com outros animais faz com que os bezerros reduzam as respostas à contenção e aumentem os comportamentos lúdicos (Vieira \& Shields, 2016). Entretanto, uma desvantagem desse tipo de alojamento é a possibilidade do desenvolvimento de mamada cruzada (Bittar, 2016).

\section{Comportamentos em bovinos}

Denomina-se comportamento tudo aquilo que se consegue perceber das reações de um animal, seja os movimentos da totalidade ou de parte de seu corpo ou mesmo a inatividade ao ambiente que o cerca (Donald Maurice Broom, 2006; Faria et al., 2011).

Carvalho et al. (2012) discutem sobre a importância de se conhecer os comportamentos dos animais. Para os autores, a partir desse conhecimento os produtores conseguirão identificar os desvios de comportamento e dessa forma adequar os sistemas minimizando o desconforto. Além disso, o potencial dos animais poderá ser explorado com maior eficiência, respeitando suas necessidades e, consequentemente, aumentando a produtividade do sistema de produção. 
Adotar práticas de bem-estar e aplicar boas práticas de manejo é necessário para promover melhores condições aos animais e aumentar sua produtividade nas propriedades rurais (Azevedo et al., 2014; Oliveira et al., 2013). Para definir a condição de bem-estar animal deve-se seguir uma visão que aborde criteriosamente o animal e o ambiente onde ele está inserido (Broom \& Fraser, 2010). De acordo com Broom \& Fraser (2010), o comportamento é uma das formas mais utilizadas para determinação do bemestar. Os animais possuem diferentes necessidades, algumas em particular, tais como água ou calor, outras associadas a sentimentos chamadas de experiências subjetivas. Outras necessidades são obtidas pela observação de anormalidades comportamentais ou fisiológicas, indicando que suas necessidades não foram satisfeitas (Broom \& Molento, 2004).

Em um sistema de produção de ruminantes é de fundamental importância que os criadores conheçam os comportamentos normais da espécie. Para tal, faz-se necessário compreender como se dão às distintas interações dos animais no ambiente onde são criados, pois esse entendimento permitirá evitar situações negativas que podem resultar em estresse e prejuízos econômicos (Malafaia et al., 2011).

Sabe-se que bovinos são animais de hábito gregário, apresentam organização social bem estabelecida e são animais com comportamento de presa (Grandin, 1997). Além dessas características, os bovinos apresentam outros padrões comportamentais e fisiológicos próprios da espécie, como a ruminação (Stricklin \& Kautz-Scanavy, 1984). Nesse sentido, Broom \& Molento (2004) discutem sobre a importância da instalação na criação de bezerros. Segundo os autores, bezerros quando criados em baias ou espaços muito pequenos, tentam limpar-se exaustivamente, porém esse comportamento torna-se impossível devido à restrição de espaço no lugar os quais foram inseridos.

Os bovinos quando são submetidos a situações que provocam dor, isolamento social e medo, apresentam estresse e reagem a essas situações aversivas, modificando o seu comportamento, podendo aumentar a sua movimentação ou tentativa de fuga (Grandin \& Deesing, 2014; Lanier et al., 2000). Assim, as instalações que restringem de forma severa a possibilidade de locomoção dentro da mesma, levam o animal apresentar bem-estar pobre (Broom \& Molento, 2004). De acordo com os autores, os animais passam apresentar comportamentos anormais, como estereotipias, automutilação e comportamentos agressivos que podem indicar que o indivíduo se encontra em condições de bem-estar pobre. Para Zanella (1995), o comportamento anormal pode ser dividido em três categorias: destrutivos, comportamento estereotipado ou apatia.

Os comportamentos destrutivos como sendo aqueles que causam lesões nos animais, tais como: as mordeduras e os vícios de sucção da cauda, orelha, flanco e vulva, bem como os comportamentos agressivos (Paranhos da Costa, 2004; Zanella, 1995). Com relação ao comportamento estereotipado, Dantzer (1991) e Zanella (1995) o definem pelo ato do animal repetir uma ação de forma invariável por longo período de tempo sem aparente função, sendo mais frequente em animais confinados.

Enquanto que o comportamento apático é aquele em que o animal apresenta inatividade excessiva, ocorre quando o ambiente não é estimulante (Broom, 1991). Para o autor, esse tipo comportamental indica que o indivíduo em questão está com dificuldade em lidar com o ambiente.

\section{Enriquecimento ambiental}

O termo enriquecimento implica em melhorias, sejam elas físicas, sociais, alimentares entre outras, aplicadas como forma de alterar de modo favorável ao ambiente (Newberry, 1995). Ambientes enriquecidos podem melhorar o bem-estar permitindo a expressão de comportamentos específicos da espécie, afetando o desenvolvimento físico e psicológico do animal (Foppa et al., 2014).

As atividades de enriquecimento ambiental devem ser pouco previsíveis e bastante motivadoras, apresentando-se como uma excelente ferramenta de redução do estresse em animais confinados, contribuindo para o desenvolvimento fisiológico e produtivo dos mesmos (Meyer et al., 2010). Muitas são as possibilidades de objetos utilizados como enriquecimento ambiental para animais confinados e, tal variação pode fomentar preferências por parte dos animais retardando a perda de interesse pelos objetos, e para tanto, os testes de aversão ou preferência por um objeto de enriquecimento ambiental são de grande importância (Volpato, 2007). O ambiente em que vivem os animais colabora parcialmente com o desenvolvimento cognitivo dos mesmos. Estes ambientes precisam ser ricos em diferentes tipos de estímulos, que podem ser táteis, visuais, olfativos e também nutricionais (Horvath et al., 2017). 
Segundo vários autores (Almeida et al., 2008; Campos et al., 2010; Gonçalves et al., 2010; et al., 2015), as técnicas de enriquecimento ambiental podem ser do tipo: Físico, quando está relacionado à estrutura física do recinto, ou seja, ao ambiente onde os animais estão inseridos. O objetivo é deixar o recinto mais semelhante ao habitat natural. Sensorial, consiste em explorar os cinco sentidos dos animais. O objetivo é aumentar o forrageamento, ou seja, a busca por alimento. Cognitivo, que consiste em dispositivos mecânicos ("quebra-cabeças") para os animais manipularem de forma que estimule suas capacidades intelectuais. Alimentar, que consiste em promover variações na alimentação dos animais, fazendo com que tenham certa dificuldade em obter o alimento. Além dessas técnicas há também o enriquecimento social, que consiste na interação intraespecífica ou interespecífica que pode ser criada dentro de um recinto (Almeida \& Monteiro Filho, 2008). Os objetivos do enriquecimento são: aumentar a diversidade e o número de comportamentos normais da espécie; reduzir a frequência de comportamentos anormais; aumentar a utilização positiva do ambiente e aumentar a habilidade de lidar com dificuldades de uma maneira mais natural (Szokalski et al., 2012).

Um problema frequente nos sistemas de criação coletivo é a ocorrência de mamada cruzada entre os animais, que é um comportamento anormal. Uma forma de reduzir esse comportamento é oferecer a eles uma maneira de expressar esse comportamento de sugar, que é natural nesses animais (Silva, 2018). Ude et al. (2011) utilizaram bico de mamadeira como enriquecimento ambiental e obtiveram resultados positivos na redução da ocorrência de mamada cruzada entre bezerros criados em grupos.

\section{Considerações finais}

Visto que a forma como os animais são manejados implicará no índice de produtividade, é importante que haja conhecimento por parte do produtor sobre os comportamentos dos animais de modo que possa avaliar mudanças comportamentais que venham comprometer a saúde dos mesmos e consequentemente a qualidade da produção.

Com isso, faz necessário por parte dos produtores a busca por melhores formas de manejo que objetivem o bom bem-estar animal.

\section{Referências bibliográficas}

Almeida, A. M. R., Margarido, T. C. C., \& Monteiro Filho, E. L. A. (2008). Influência do enriquecimento ambiental no comportamento de primatas do gênero Ateles em cativeiro. Arquivos de Ciências Veterinárias e Zoologia da UNIPAR, 11(2), 97-102.

Almeida, L. S. P., Lobato, J. F. P., \& Schenkel, F. S. (2002). Data de desmame e desempenho reprodutivo de vacas de corte. Revista Brasileira de Zootecnia, 31(3), 1223-1229. DOI: https://doi.org/10.1590/s1516-35982002000500019

Alvim, M. J., Paciullo, D. S. C., Carvalho, M. M., Aroeira, L. J. M., Carvalho, L. A., Novaes, L. P., Gomes, A. T., Miranda, J. E. C., \& Ribeiro, C. C. L. (2005). Sistema de produção de leite com recria de novilhas em sistemas silvipastoris. Embrapa Gado de Leite, 1(1), 1-10.

Azevedo, R. A., Rufino, S. R. A., Duarte, D. V. L., Soares, A. C. M., \& Geraseev, L. C. (2014). Desempenho de bezerros leiteiros em aleitamento artificial convencional ou fracionado. Revista Brasileira de Saúde e Produção Animal, 15(1), 237-247. DOI: https://doi.org/10.1590/s1519$\underline{99402014000100023}$

Bittar, C. M. M. (2016). Instalações para bezerras leiteiras. Cadernos Técnicos de Veterinária e Zootecnia, 81, 26-44.

Broom, D. M. (1986). Indicators of poor welfare. British Veterinary Journal, 142(6), 524-526. DOI: https://doi.org/10.1016/0007-1935(86)90109-0

Broom, D. M. (1991). Animal welfare: concepts and measurement. Journal of Animal Science, 69(10), 4167-4175. DOI: https://doi.org/10.2527/1991.69104167x

Broom, D. M, \& Molento, C. F. M. (2004). Animal welfare: concept and related issues-review. Archives of Veterinary Science, 9(2), 1-11.

Broom, D. M, \& Fraser, A. F. (2010). Comportamento e bem-estar de animais domésticos. Editora Manole. 
Broom, Donald Maurice. (2006). Behaviour and welfare in relation to pathology. Applied Animal Behaviour Science, 97(1), 73-83. DOI: http://dx.doi.org/10.1016/j.applanim.2005.11.019

Burguer, P. J., Pereira, J. C., Queiroz, A. C., Silva, J. F. C., Valadares, S. C., Cecon, P. R., \& Casali, A. D. P. (2000). Comportamento ingestivo de bezerros holandeses alimentados com dietas contendo diferentes níveis de concentrado. Revista Brasileira de Zootecnia, 29(1), 236-242. DOI: http://dx.doi.org/10.1590/S1516-35982000000100031

Busch, G., Weary, D. M., Spiller, A., \& von Keyserlingk, M. A. G. (2017). American and German attitudes towards cow-calf separation on dairy farms. PloS One, 12(3), e0174013. DOI: https://doi.org/10.1371/journal.pone.0174013

Campos, J. A., Tinôco, I. F. F., Silva, F. F., Pupa, J. M. R., \& Silva, I. J. O. (2010). Enriquecimento ambiental para leitões na fase de creche advindos de desmame aos 21 e 28 dias. Revista Brasileira de Ciências Agrárias, 5(2), 272-278. DOI: https://doi.org/10.5039/agraria.v5i2a660

Campos, O. F., \& Campos, A. T. (2004). Instalações para bezerros de rebanhos leiteiros. Embrapa Gado de Leite-Circular Técnica, 1, 1-6.

Carneiro, J. C. (2015). Comportamento alimentar e social de bezerras leiteiras F1 Holandês x Gir durante a fase de aleitamento e desaleitamento. Universidade Federal de Minas Gerais.

Carvalho, W. T. V., Villanova, D. F. Q., Minighin, D. C., Lima, W. B. G., Matos Teixeira, A., \& Pereira, R. V. G. (2012). Comportamento de bezerras da raça Holandesa alimentadas com silagem pré-secada do capim Tifton 85 (Cynodon ssp.). PUBVET, 12(2), 1-7. DOI: https://doi.org/10.22256/pubvet.v12n2a39.1-7

Costa, L. S., Pereira, D. F., Bueno, L. G. F., \& Pandorfi, H. (2012). Some aspects of chicken behavior and welfare. Brazilian Journal of Poultry Science, 14(3), 159-164. DOI: https://doi.org/10.1590/s1516-635x2012000300001

Dantzer, R. (1991). Stress, stereotypies and welfare. Behavioural Processes, 25(2-3), 95-102. DOI: https://doi.org/10.1016/0376-6357(91)90012-o

Façanha, D. A. E., Vasconselos, Â. M., Chaves, D. F., Silva, W. S. T., Morais, J. H. G., \& Olivo, C. J. (2011). Respostas comportamentais e fisiológicas de bezerros leiteiros criados em diferentes tipos de instalações e dietas líquidas. Acta Veterinaria Brasilica, 5(3), 250-257.

Faria, L. A. N., Barbosa, O. R., Zeoula, L. M., Aguiar, S. C., Prado, R. M., \& Bertolini, D. A. (2011). Produto à base de própolis (LLOS) na dieta de bovinos inteiros confinados: comportamento animal e respostas sanguíneas. Acta Scientiarum. Animal Sciences, 33(1), 79-85. DOI: https://doi.org/10.4025/actascianimsci.v33i1.10671

FAWC. (2009). Farm animal welfare in Great Britain: Past, present and future. Farm Animal Welfare Council.

Filipini, B., Dantas, A., \& Montanha, A. A. O. (2016). Bem-estar e comportamento de ovinos em sistema intensivo. Enciclopéia Biosfera, 13(24), 1-15. DOI: https://doi.org/10.18677/encibio_2016b_014

Foppa, L., Caldara, F. R., Machado, S. P., Moura, R., Santos, R. K. S., Nääs, I. A., \& Garcia, R. G. (2014). Enriquecimento ambiental e comportamento de suínos: revisão/ environmental enrichment and behaviour of pigs. Revista Brasileira de Engenharia de Biossistemas, 8(1), 1-7. DOI: https://doi.org/10.18011/bioeng2014v8n1p1-7

Fraser, A. F., \& Broom, D. M. (1997). Farm animal behaviour and welfare (Issue Ed. 3). Cab International.

Galindo, F., Broom, D. M., \& Jackson, P. G. G. (2000). A note on possible link between behaviour and the occurrence of lameness in dairy cows. Applied Animal Behaviour Science, 67(4), 335-341. DOI: http://dx.doi.org/10.1016/S0168-1591(99)00114-8

Gomes, V., \& Madureira, K. M. (2016). Sanidade na criação de bezerras: dos 31 dias ao desmameParte III.

Gonçalves, M. A. B., Silva, S. L., Tavares, M. C. H., Grosmann, N. V., F., C. C., \& Di Castro, P. H. G. (2010). Comportamento e bem-estar animal: o Enriquecimento Ambiental. In A. Andrade, M. C. R. Andrade, A. M. Marinho, \& J. Ferreira Filho (Eds.), Biologia, Manejo e Medicina de primatas nãohumanos na pesquisa Biomédica. FIOCRUZ. 
Grandin, T. (1997). Assessment of stress during handling and transport. Journal of Animal Science, 75(1), 249-257. http://www.journalofanimalscience.org/content/75/1/249.abstract

Grandin, Temple, \& Deesing, M. J. (2014). Genetics and behavior during handling, restraint, and herding. In Temple Grandin \& M. J. Deesing (Eds.), Genetics and the Behavior of Domestic Animals (Second, Vol. 1, pp. 115-158). Academic Press. DOI: http://dx.doi.org/10.1016/B978-0-12-394586-0.00004-4

Horvath, K., Fernandez, M., \& Miller-Cushon, E. K. (2017). The effect of feeding enrichment in the milk-feeding stage on the cognition of dairy calves in a T-maze. Applied Animal Behaviour Science, $187,8-14$.

Hötzel, M. J., \& Machado Filho, L. C. P. (2004). Bem-estar animal na agricultura do século XXI. Revista de Etologia, 6(1), 3-15.

Jasper, J., \& Weary, D. M. (2002). Effects of ad libitum milk intake on dairy calves. Journal of Dairy Science, 85(11), 3054-3058.

Kondo, S., \& Hurnik, J. F. (1988). BEHAVIORAL AND PHYSIOLOGICAL RESPONSES TO SPATIAL NOVELTY IN DAIRY COWS. Canadian Journal of Animal Science, 68(2), 339-343. DOI: https://doi.org/10.4141/cjas88-038

Lanier, J. L., Grandin, T., Green, R. D., Avery, D., \& McGee, K. (2000). The relationship between reaction to sudden, intermittent movements and sounds and temperament. Journal of Animal Science, 78(6), 1467-1474.

Lauber, M. C. Y., Hemsworth, P. H., \& Barnett, J. L. (2006). The effects of age and experience on behavioural development in dairy calves. Applied Animal Behaviour Science, 99(1-2), 41-52. DOI: http://dx.doi.org/10.1016/j.applanim.2005.10.009

Malafaia, P., Barbosa, J. D., Tokarnia, C. H., \& Oliveira, C. M. C. (2011). Distúrbios comportamentais em ruminantes não associados a doenças: origem, significado e importância. Pesquisa Veterinária Brasileira, 31(9), 781-790.

Mendl, M. (2001). Avaliando o estado de bem-estar. Nature, 410, 31-32.

Meyer, S., Puppe, B., \& Langbein, J. (2010). Cognitive enrichment in zoo and farm animals-implications for animal behaviour and welfare. Berliner Und Munchener Tierarztliche Wochenschrift, 123(11-12), 446-456.

Miranda-de la Lama, G. C., Villarroel, M., \& María, G. A. (2012). Behavioural and physiological profiles following exposure to novel environment and social mixing in lambs. Small Ruminant Research, 103(2-3), 158-163. DOI: http://dx.doi.org/10.1016/j.smallrumres.2011.08.007

Miranda, J. E. C., Ribeiro A.C., Campos, O. F., \& Novaes, L. P. (2003). Cria e recria de fêmeas leiteiras: passo a passo. Embrapa Gado de Leite-Comunicado Técnico, 1, 1-6.

Moura, I. C. F., Kuss, F., Moletta, J. L., Menezes, L. F. G., Henrique, D. S., Paris, M., \& Cullmann, J. R. (2014). Desempenho de bezerros de corte Purunã submetidos a diferentes sistemas de desmame. Semina: Ciências Agrárias, 35(4), 2711-2721.

Newberry, R. C. (1995). Environmental enrichment: increasing the biological relevance of captive environments. Applied Animal Behaviour Science, 44(2-4), 229-243.

Oliveira, A. A., \& Azevedo, H. C. (2005). Criação de bezerras em sistemas de produção de Leite. Embrapa Tabuleiros Costeiros. Circular Técnica, 1-6.

Oliveira, A. P. G., Costa, W. M., Costa, W. M., Nunes, R. A., Dias, N. C. S., \& Madella-Oliveira, A. F. (2015). Influência do enriquecimento ambiental nos padrões de comportamentos sociais e anormais de cabras em confinamento. Archives of Veterinary Science, 20(2), 1-7.

Oliveira, C. E. A., Brando, P. T. V, Carneiro Filho, A. J., \& Mingote, L. C. (2014). Criação e Desenvolvimento de Bezerras Leiteiras no Período de Aleitamento: Práticas de Manejo. Instituto de Ciências Da Saúde, Agrárias e Humanas, 1-57.

Oliveira, D. P., Oliveira, M. V. M., Vargas Júnior, F. M., Luz, D. F., Simões, A. R. P., Oliveira, C. A. L., Braga Netto, A. L., \& Silva, S. C. C. (2013). Desempenho de bezerros leiteiros lactentes alimentados com feno. Archivos de Zootecnia, 62(239), 357-367.

Paranhos da Costa, M., \& Cromberg, V. U. (1997). Alguns aspectos a serem considerados para melhorar 
o bem-estar de animais em sistemas de pastejo rotacionado. In A. M. Peixoto, J. C. Moura, \& V. C. Faria (Eds.), Fundamentos do pastejo rotacionado. FEALQ: Piracicaba (pp. 273-296). FEALQ.

Paranhos da Costa, M. J. R. (2004). Comportamento e bem-estar de bovinos e suas relações com a produção de qualidade. Anais Dos Simpósios Da 4la Reunião Da Sociedade Brasileira de Zootecnia, 260-268.

Passillé, A. M., Marnet, P.-G., Lapierre, H., \& Rushen, J. (2008). Effects of twice-daily nursing on milk ejection and milk yield during nursing and milking in dairy cows. Journal of Dairy Science, 91(4), $1416-1422$.

Pellegrini, L. G., Restle, J., Neumann, M., \& Souza, A. N. M. (2006). Desempenho de bezerros desmamados precocemente, mantidos em pastagem de capim elefante (Pennisetum purpureum, Schum), com diferentes níveis de suplementação. Revista Brasileira de Zootecnia, 36(6), 18831889.

http://www.scopus.com/inward/record.url?eid=2-s2.033845398198\& partnerID=40\&md5=b5ff4a2e802033584b695097d372bd95

Prado, I. N., \& Von Tiesenhauser, I. M. E. V. (1987). Desempneho de bezerros submetidos ao desmame precoce e alimentados com sucedâneos à base de soja, enriquecido ou não com gordura de porco. Revitsa Unimar, 9(1), 123-131.

Prado, I. N., \& Von Tiesenhauser, I. M. E. V. (1988). Uso de sucedâneo de leite, à base de soja, acrescido ou não de gordura de porco, no aleitamento de bezerros. Revista UNIMAR, 10(1), 39-47.

Restle, J., Pacheco, P. S., Moletta, J. L., Brondani, I. L., \& Cerdótes, L. (2003). Grupo genético e nível nutricional pós-parto na produção e composição do leite de vacas de corte. Revista Brasileira de Zootecnia, 32(3), 585-597. DOI: http://dx.doi.org/10.1590/S1516-35982003000300010.

Rodríguez, C., Castro, N., Capote, J., Morales-delaNuez, A., Moreno-Indias, I., Sánchez-Macías, D., \& Argüello, A. (2009). Effect of colostrum immunoglobulin concentration on immunity in Majorera goat kids. Journal of Dairy Science, 92(4), 1696-1701. DOI: http://dx.doi.org/10.3168/jds.2008-1586

Santos, G. T., Bertolini, D. A., Macedo, F. A. F., Prado, I. N., \& Martins, E. N. (1994). Variabilidade em imunoglobulina $\mathrm{G}(\mathrm{IgG})$ no colostro de cabra de primeira ordenha e absorção intestinal de $\operatorname{IgG}$ pelos cabritos recém-nascidos. Brazilian Archives of Biology and Technology, 37(2), 285-292.

Santos, G. T., Grongnet, J. F., Prado, I. N., \& Lareynie, J. (1988). Efeito da ordem de lactação sobre os níveis em imunoglobulinas $\mathrm{G}$ (IgG) do colostro bovino. Revista UNIMAR, 10(1), 31-38.

Silva, M. D. (2018). Avaliação de diferentes dietas líquidas associadas ao enriquecimento ambiental no desempenho e comportamento de bezerros leiteiros. Universidade de São Paulo.

Stricklin, W. R., \& Kautz-Scanavy, C. C. (1984). The role of behavior in cattle production: A review of research. Applied Animal Ethology, 11(4), 359-390. DOI: http://dx.doi.org/10.1016/0304$\underline{3762(84) 90043-9}$

Szokalski, M. S., Litchfield, C. A., \& Foster, W. K. (2012). Enrichment for captive tigers (Panthera tigris): Current knowledge and future directions. Applied Animal Behaviour Science, 139(1-2), 1-9. DOI: http://dx.doi.org/10.1016/j.applanim.2012.02.021

Ude, G., Georg, H., \& Schwalm, A. (2011). Reducing milk induced cross-sucking of group housed calves by an environmentally enriched post feeding area. Livestock Science, 138(1-3), 293-298.

Vieira, F., \& Shields, E. S. (2016). Bem-estar na produção de bezerras leiteiras-Parte II.

Vieira, F. V. R., \& Silva, I. J. O. (2014). Aspectos críticos da criação de bezerros leiteiros no Brasil: Ponto de vista do bem-estar animal.

Volpato, G. L. (2007). Considerações metodológicas sobre os testes de preferência na avaliação do bemestar em peixes. Revista Brasileira de Zootecnia, 36, 53-61.

Williams, G. L. (2005). Fisiologia e manejo reprodutivo de vacas de corte pós-parto. Curso Novos Enfoques Na Produção e Reprodução de Bovinos, 9, 195-203.

Zanella, A. J. (1995). Indicadores fisiológicos e comportamentais do bem-estar animal. A Hora Veterinária, 14(8), 47-52.

Recebido: 20 de março, 2020.

Aprovado: 20 de maio, 2020.

Disponível online: 17 de agosto, 2020.

Licenciamento: Este artigo é publicado na modalidade Acesso Aberto sob a licença Creative Commons Atribuição 4.0 (CC-BY 4.0), a qual permite uso irrestrito, distribuição, reprodução em qualquer meio, desde que o autor e a fonte sejam devidamente creditados. 